\title{
Editorial: Potential Sensors for the Forthcoming 6G/loE - Electronics and Physical Communication Aspects
}

\author{
Fadi Al-Turjman ${ }^{1}$ \\ Published online: 6 October 2020 \\ (C) Springer Science+Business Media, LLC, part of Springer Nature 2020
}

\section{Editorial:}

The new $6 \mathrm{G}$ and Internet of Everything $(6 \mathrm{G} / \mathrm{IoE})$ infrastructure is expected to transform the world of connected sensors and reshape industries. Such a revolution would of course require research and development for the co-existence and device inter-operability of sensors' physics/electronics with $6 \mathrm{G}$ networks, novel sensor prototypes, deployment, placement, control and management strategies, smart and intelligent network on chip (NoC) solutions, energy-efficient sensing materials with the support of new infrastructures, development of new energy harvesting methods in promising electronics sensors, inter/intra communication protocols and standards, integration of sensors with the existing Cloud-based infrastructures using 6G, and physical circuits reliability/ privacy and security issues for sensors employing 6G. Such research would also require case studies and physical deployment of sensors for the emerging IoE paradigm in $6 \mathrm{G}$ networks, studies using test beds, experimental results, as well as various performance evaluation and modelling approaches. Understanding the capabilities associated with these potential sensors is essential for practical implementation as they help modernize and streamline the motivating electronics and physical communication techniques in the near future. Consequently, researchers, scientists, and engineers face emerging challenges in designing sensor-based systems that can efficiently be integrated with the $6 \mathrm{G} / \mathrm{IoE}$ communication paradigms.

This special issue features nine selected papers with high quality. The first article, "Using Multiple RPL Instances to Enhance the Performance of New 6G and Internet of Everything (6G/IoE)-based Healthcare Monitoring Systems", investigates the use of multiple RPL instances in healthcare monitoring systems to enhance the $6 \mathrm{G} / \mathrm{IoE}$ network

Fadi Al-Turjman

fadi.alturjman@antalya.edu.tr

1 Near East University, Nicosia, Mersin 10, Turkey

performance. The authors evaluate the performance of their proposed approach using Cooja simulator in terms of two key routing performance metrics: average Packet Delivery Ratio (PDR) and average latency. The results of the simulation showed that, by using the proposed approach, the performance of healthcare monitoring systems is enhanced in all cases, in terms of latency, from tens of seconds to less than one second.

The second article titled "Creating collision free communication in IoT with 6G using Multiple Machine Access Learning Collision Avoidance protocol" proposes a Multiple Machine Access Learning with Collision Carrier Avoidance (MMALCCA) protocol in the environment of $6 \mathrm{G}$ Internet of Things for creating an effective communication process. This protocol employs the Media Access Control (MAC) protocol for the sync of high-speed wireless communication networks in the Terahertz (THz) band. MMALCCA performs multiple machine access and collision control for improving the resource utilization and latency-less services of the users. The decisions of the protocol are made using the output of the classification and regression learning method for improving the efficiency of MAC sync. The performance of the proposed protocol is verified using the metrics latency, collision probability, service failure, and resource utilization by varying channels and user equipment density. The proposed MMALCCA achieves $20.09 \%$ less latency, $6.77 \%$ less collision probability, $13.32 \%$ fewer service failures, and improves resource utilization by $8.34 \%$ for the varying UEs. For the varying channels, the proposed protocol achieves $7.49 \%$ high resource utilization and reduces latency, collision probability, and service failure by $25.21 \%, 8.57 \%$, and $13.7 \%$, respectively.

In the next article with the title "CNN-based Occlusionaware Vehicle detection in urban traffic sensing systems", authors present a new occlusion-aware vehicle detection CNN framework, which is an effective and efficient framework for the vehicle detection systems in the IoE era. First, authors concatenate the low-level and high-level feature maps to capture more robust feature representation, then they fuse the 
local and global feature maps for handling vehicle occlusion, and the context information has also been adopted in their framework. Extensive experiments demonstrate the competitive performance of the proposed framework. The proposed methods achieve better effect than primal Faster R-CNN in terms of accuracy on a new urban traffic surveillance dataset (UTSD) which contains a mass of occlusion vehicles and complex scenes.

The fourth article titled "The Effect of Mobile Wearable Waist Assist Robot on Lower Back Pain during Lifting and Handling Tasks", a mobile, flexible, and wearable waist assist device, using pneumatic artificial muscles has been developed for the smart IoE environments. A novel study relevant to potential sensors/devices with their physical aspects that can help in realizing the IoE in practice was proposed. Novel sensor prototypes, deployment, placement, control and management have been discussed and validated. The paper shows how physical sensing techniques can enable a participatory approach for achieving integrated solutions and creating novel applications related to smart environments. With the proliferation of such intelligent devices, sensors are expected to lead further innovation in IoE.

Fiber Bragg Grating (FBG) is achieved by refractive index modulation in evolving $6 \mathrm{G} / \mathrm{IoE}$ smart environments in order to measure temperature and strain. However, there are a few types of conventional refractive index modulation methods, and a few types of echo spectrum. The fifth article, "Fiber Bragg Grating Sensor Based on Refractive Index Segment Code of mobile Modulation", proposes an index code modulation based on an Industrial Internet of Things (IIoT) method. The FBG is divided into a plurality of interval segments. The spectral shape of the echo is controlled by encoding and modulating each interval. When the segment size satisfies some requirements, the positive and negative modes of the sub-FBG are calculated by the coupled-mode theory, and then the mode functions of each segment are solved by the matrix transmission algorithm. The equivalent function of the reflectance distribution field is obtained. According to the relevant parameters of FBG, the mathematical model of the FBG modulation spectral distribution has been established by the refractive index modulation method. Through the simulation analysis of the fractional refractive index modulation, the results show that the echo spectral peak, half-width, etc. have a larger tunable range. In the experiment, a femtosecond laser was used to accurately encode and modulate the refractive index, and two different types of segmented FBGs were fabricated according to design requirements. The experimental results show that the design of different segmented structures has a significant impact on the spectral distribution of the echo.

On the other hand, existing and utilized networks security are not fully considering the randomness of attacks and the accuracy of the prediction of attack intentions. Therefore, the sixth article, titled "Network Security Situation Prediction of improved Lanchester Equation Based on Time Action Factor", proposes a network behavior calculation model based on the Lanchester equation of the time action factor. This paper uses smooth differential manifold homeomorphic transformation to define network behavior, defines the calculation method of the behavior utility based on the principle of differential geometry, that combines the second linear law of the Lanchester equation and the square law, and uses the time action factor to provide the active defense. The proposed simulation results show that the model can be used to analyze the network offensive and defensive process and can effectively predict the network offensive and defensive results under both active and passive modes.

The Internet of Everything (IoE) provides a platform that allows devices to be remotely connected, sensed, and controlled across the network infrastructure. The smart home in the era of the IoE is born on the basis of the high integration of emerging communication technologies such as big data, sensors, and machine learning. In the seventh article, titled "Indoor WLAN Personnel Intrusion Detection Using Transfer Learning-aided Generative Adversarial Network with Light-loaded Database" the authors focus on wireless detection technologies using smartphones and computers in smart homes. Among them, the indoor Wireless Local Area Network (WLAN) personnel intrusion detection technology based on the database construction has become one of the comprehensive detection technologies by advantages of the convenient accessibility of the WLAN signal and minimal hardware requirement. However, the considerable labor and time cost involved in the database construction affects the popularity and application of database-based intrusion detection systems. To cope with this problem, authors propose a new indoor WLAN personnel intrusion detection approach with the reduced overhead of the database construction. Specifically, first of all, the offline database is extended by fake Received Signal Strength (RSS) data, which are generated by the Generative Adversarial Network (GAN) based supervised learning from actual labeled RSS data. Second, the difference between the extended database and online RSS data caused by the time-variant environment noise is reduced by minimizing the Maximum Mean Discrepancy (MMD) between marginal distributions of RSS data through the transfer learning. Finally, the intrusion detection is achieved by classifying online RSS data with classifiers trained from the extended database. Furthermore, experimental results show that the proposed approach can not only perform well in reducing the database overhead and the difference of data in source and target domains, which are corresponding to the same environment state but also detect environment states with satisfactory accuracy.

Meanwhile, incentives are very important to be employed in any defensive mechanism against DDoS attack. Incentive is a major concept abandoned by most of the defensive 
mechanisms that have been proposed so far. It is a tool that can motivate users to send data wisely into the network. Another article, titled "A Two-tier Multi Attribute Based Truthful Auction Mechanism for Mitigating DDoS Attacks" proposes a two layered multi-attribute auction mechanism for incentivizing users by imposing payment schemes as well as by providing rewards. Apart from this, authors have developed a reputation assessment procedure to identify malicious user by monitoring his credibility score calculated through his marginal utility. Identified malicious users are then mapped to different levels of suspiciousness. Identified legitimate users are forwarded towards first level of auction in which virtual users have been added by service provider to increase the competition among users. Critical values are computed for every user and the users satisfying the criteria are moved towards the second level. In second level, greedy method is utilized for resource allocation. Extensive simulations have been conducted on Matlab to check the validity of the proposed model. Rate of social welfare degradation and user's satisfaction are utilized to check the appropriateness and validity of the model. Results from experimentation have shown that proposed model is able to generate enough revenue for the service provider and is able to provide acceptable QoS to identified legitimate users when there is an increase in number of malicious users.

The last article titled "An Improved IDAF-FIT Clustering Based ASLPP-RR Routing with Secure Data Aggregation in Wireless Sensor Network" investigated the integration of Distributed Autonomous Fashion with Fuzzy Ifthen Rules (IDAF-FIT) algorithm for clustering, and Cluster Head $(\mathrm{CH})$ election. To transmit the data packet from the source to the destination node while choosing an optimal path, the routing concept has been maintained. For this purpose, an Adaptive Source Location Privacy Preservation Technique using Randomized Routes (ASLPP-RR) is presented for routing. Also, Secure Data Aggregation based on Principle Component Analysis (SDA-PCA) algorithm is performed with end-to-end confidentiality and integrity. Finally, the security of confidential data is analyzed properly to obtain a better result than the existing approaches. The overall perfor- mance of the proposed methodology when compared with existing is expressed in terms of $20 \%$ higher packet delivery ratio, $15 \%$ lower packet dropping ratio, $18 \%$ higher residual energy, 22\% higher network lifetime, and 16\% lower energy consumption.

Acknowledgements The guest editors are thankful to the anonymous reviewers for their effort in reviewing the manuscripts. We are also thankful to the Edit-in-Chief, Dr. Imrich Chlamtac for his supportive guidance during the entire process.

Publisher's note Springer Nature remains neutral with regard to jurisdictional claims in published maps and institutional affiliations.

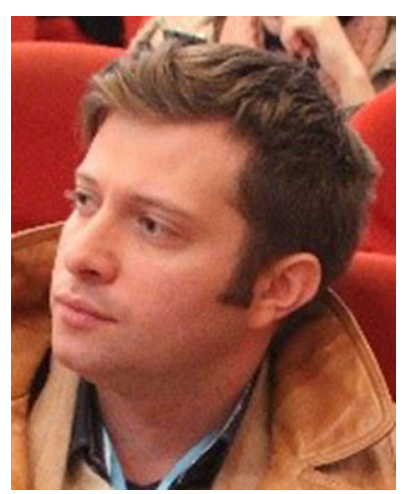

Prof. Dr. Fadi Al-Turjman received his Ph.D. in computer science from Queen's University, Kingston, Ontario, Canada, in 2011. He is a full professor and a research center director at Near East University, Nicosia, Cyprus. Prof. Al-Turjman is a leading authority in the areas of smart/intelligent, wireless, and mobile networks' architectures, protocols, deployments, and performance evaluation. His publication history spans over 250 publications in journals, conferences, patents,

books, and book chapters, in addition to numerous keynotes and plenary talks at flagship venues. He has authored and edited more than 25 books about cognition, security, and wireless sensor networks' deployments in smart environments, published by Taylor and Francis, Elsevier, and Springer. He has received several recognitions and best papers' awards at top international conferences. He also received the prestigious Best Research Paper Award from Elsevier Computer Communications Journal for the period 2015-2018, in addition to the Top Researcher Award for 2018 at Antalya Bilim University, Turkey. Prof. Al-Turjman has led a number of international symposia and workshops in flagship communication society conferences. Currently, he serves as an associate editor and the lead guest/associate editor for several well reputed journals, including the IEEE Communications Surveys and Tutorials(IF 23.9) and the Elsevier Sustainable Cities and Society (IF 5.7). 